\title{
MiR-29b Downregulation Induces Phenotypic Modulation of Vascular Smooth Muscle Cells: Implication for Intracranial Aneurysm Formation and Progression to Rupture
}

\author{
Liqian Sun ${ }^{a}$ Manman Zhao Jingbo Zhang ${ }^{\text {a }}$ Ming Lva Youxiang Lia Xinjian Yang \\ Zhongxue $\mathrm{Wu}^{\mathrm{a}}$ Aihua $\mathrm{Liu}^{\mathrm{a}}$
}

aDepartment of Interventional Neuroradiology, Beijing Neurosurgical Institute and Beijing Tiantan Hospital, Capital Medical University, Beijing, bDepartment of Histology and Embryology, School of Basic Medical Science, North China University of Science and Technology, Hebei, Tangshan, China

\section{Key Words}

MiR-29b - Intracranial aneurysm • Vascular smooth muscle cell • Phenotypic modulation • ATG14 • Autophagy

\begin{abstract}
Background/Aims: Our previous microarray results identified numerous microRNAs (miRNAs), including miR-29b, that were differentially expressed in the serum of intracranial aneurysm (IA) patients. The current study aimed to investigate whether miR-29b downregulation in IA could promote the phenotypic modulation of vascular smooth muscle cells (VSMCs) involved in the pathogenesis of aneurysm by activating ATG14-mediated autophagy. Methods: First, the levels of miR-29b and autophagy related genes (ATGs) between IA patients and normal subjects were compared. Next, we modified the level of miR-29b via lentivirus particles in the VSMCs and examined the effects of miR-29b on proliferation, migration, and phenotypic modulation of VSMCs from a contractile phenotype to a synthetic phenotype, as well as the levels of autophagy. Finally, the binding of miR-29b to the 3'UTR of ATG14 mRNA and its effects on ATG14 expression were analysed by a luciferase reporter assay and Western blot, respectively. Results: The level of miR-29b was decreased, and autophagy markers were increased in the IA patients compared to that of the normal subjects. Knockdown of miR-29b significantly promoted VSMCs proliferation and migration and, more importantly, induced the phenotypic modulation associated with autophagy activation, whereas miR-29b overexpression showed the opposite effects. The luciferase reporter assay demonstrated that ATG14 was a functional target gene of miR-29b. Notably, knockdown of ATG14 by siRNA apparently abrogated miR29b inhibition-mediated phenotypic modulation. Conclusion: Downregulation of miR-29b induced VSMCs phenotypic modulation by directly activating ATG14-mediated autophagy, which is associated with the formation, growth and rupture of IAs.




\section{Cellular Physiology Cell Physiol Biochem 2017;41:510-518

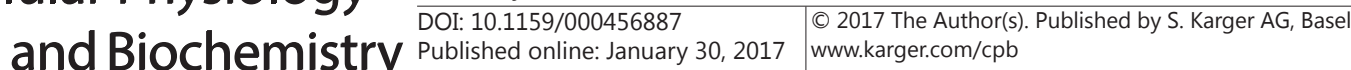 \\ Sun et al.: MiR-29b Downregulation Induces Phenotypic Modulation in VSMCs}

\section{Introduction}

Intracranial aneurysms (IAs) are important cerebrovascular diseases with a prevalence of $1-5 \%$, and the rupture of IAs causes a life-threatening subarachnoid haemorrhage (SAH), with an acute mortality rate of approximately 50\% [1]. The risk of IA rupture elevates with increasing aneurysm size and aneurysm wall irregularities. IA is characterized by a loss of the integrity of arterial walls including endothelium dysfunction, intimal hyperplasia, disorganized extracellular matrix (ECM) and inflammation [2]. Moreover, VSMCs play a fundamental role in blood pressure regulation and display remarkable plasticity under various disease conditions [3]. In response to environmental stimuli, VSMCs can undergo profound changes in phenotype, switching from a contractile state into a pro-inflammatory and matrix remodelling phenotype, termed synthetic VSMCs [4]. Accumulating data have suggested that VSMCs phenotypic modulation is involved in the pathogenesis of IA formation and rupture [4-6]. However, the genetic mechanisms underlying these VSMCs alterations are not yet fully understood.

MicroRNAs (miRNAs), non-coding short RNA molecules composed of 20-25 nucleotides, negatively regulate gene expression at the post-transcriptional level by binding to the 3'UTR region of target mRNAs, thereby inhibiting translation or inducing mRNA degradation [5]. Prior studies have noted that miRNAs play a critical role in vascular diseases through regulating VSMCs and endothelial cell functions, such as proliferation, migration, apoptosis, and synthesis/secretion of ECM proteins [8-10]. Our previous microarray analyses have identified 86 serum miRNAs with significantly different expression between normal subjects and aneurysm patients, suggesting that dysregulation of miRNAs might participate in the modulation of basic physiological and pathological processes involved in IA and may be a warning sign for rupture [6]. These aberrantly expressed miRNAs are related to apoptosis, vascular remodelling, ECM degradation, inflammation and immune responses in IA walls. Importantly, dysregulated miRNAs could control VSMCs phenotypic modulation and the response to injury [7]. Among them, miR-145 has been reported to regulate VSMCs phenotype by upregulation of VSMC-specific proteins [8]. A cluster of miR-221/222 was shown to be a key player in VSMCs phenotypic and behavioural changes in response to vascular injury [9].

We have previously reported that miR-29b is significantly downregulated in the serum of IA patients compared with that of normal subjects. However, little is known about the biological significance of miR-29b in VSMCs function and phenotypic modulation. Here, the aim of the present study was to investigate the potential effects of miR-29b on VSMCs phenotypic modulation and the autophagy-related molecular mechanism involved in IA pathogenesis. Ongoing work promises to provide new targets for the diagnosis and treatment of IA.

\section{Materials and Methods}

\section{Blood and tissue samples from IA patients}

The study was approved by the Human Ethics Committee of Capital Medical University, and written informed consent was obtained from all participants. A total of 60 patients diagnosed with IA were recruited from the Department of Interventional Neuroradiology, Beijing Tiantan Hospital of Capital Medical University (Beijing, China) from October 2014 to May 2015 and divided into an unruptured group ( $\mathrm{n}=30$ ) and ruptured group $(n=30)$. The size of the aneurysm ranged from $20 \times 15 \mathrm{~mm}$ to $2.5 \times 1.5 \mathrm{~mm}$. Age-matched healthy subjects without a family history of IA were gathered as a control group $(n=30)$. The equivalent number of middle meningeal artery (MMA) segments from patients with matched sex and age were obtained during standard neurosurgical procedures (traumatic haematoma, tumour resection, IA clipping) as controls. Each group consisted of 15 males and 15 females, and the age ranged from 20 to 80 years old. There were no significant differences among the three groups in the patient's age and the size of the aneurysm $(P>0.05)$. Blood and tissue samples were collected from all subjects for assessment. 


\section{Cellular Physiology Cell Physiol Biochem 2017;41:510-518

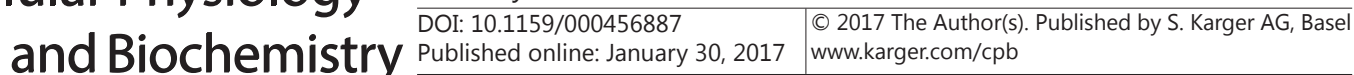 \\ Sun et al.: MiR-29b Downregulation Induces Phenotypic Modulation in VSMCs}

\section{Lentiviral vector construction and infection}

The full-length pre-miR-29b, miR-29b specific inhibitor or corresponding negative control oligonucleotide was cloned into the GV280 lentiviral vector (GeneChem, Shanghai, China) to construct a lentivirus encoding miR-29b (LV-miR-29b), lentivirus inhibiting miR-29b (LV-anti-miR-29b) and an LV-NC and LV-anti-NC, respectively. Cultured VSMCs under good culture conditions from each experimental group were infected with LV-miR-29b, LV-NC, LV-anti-miR-29b or LV-anti-NC. Three days after infection, the level of miR-144 were detected by real-time PCR.

\section{Cell proliferation assay}

The proliferation of VSMCs was determined by using 5-bromo-2-deoxy-uridine (BrdU) Labelling and manual cell counting, according to the manufacturer's instructions.

\section{Migration assay}

The migration of VSMCs was evaluated using a scratch wound healing assay and transwell migration assays as previously described [10].

\section{Luciferase reporter assays}

The sequence of segments with WT or mutant 3'-UTR of ATG14 was cloned into the lentiviral pGL3 vector (GeneChem, Shanghai, China). The pGL3- ATG14 WT or pGL3- ATG14 mut was co-transfected with miR-29b into cultured VSMCs. After $48 \mathrm{~h}$, Firefly and Renilla luciferase activity were determined by a dualluciferase reporter assay system.

\section{RNA interference (RNAi) and transfection}

For specific gene knockdown of ATG14 mRNA, the lentiviral particles of siRNA targeting ATG14 (si-ATG14) or scramble siRNA control (si-Control) were introduced into a lentiviral vector (Genechem, Shanghai, China).

RNA extraction and real-time PCR assays for miRNA and mRNA

Total RNA was extracted from VSMCs with TRIzol Reagent (Invitrogen, Carlsbad, CA, USA). Reverse transcription reactions were performed with the Superscript First Strand cDNA synthesis system (Invitrogen, CA, USA). Real-time PCR was performed with a One Step SYBR® Prime Script TM RT-PCR Kit II (Takara, Tokyo, Japan). U6 and GAPDH served as an internal control for miRNA and mRNAs assays. The data were analysed using the $2^{-\Delta \Delta t}$ method.

\section{Immunocytochemistry staining}

Slides of cells were stained in accordance with the instructions for the SABC immunocytochemistry kit (Beyotime, Shanghai, China). The slides were incubated in 5\% BSA solution for $20 \mathrm{~min}$, incubated overnight at $4^{\circ} \mathrm{C}$ with rabbit anti-LC3 (Santa Cruz Biotechnology, CA, USA, diluted 1:100), and then incubated with horseradish peroxidase-conjugated anti-rabbit IgG for $60 \mathrm{~min}$. Diaminobenzidine (Beyotime, Shanghai, China) was used to reveal the immunohistochemical reaction.

\section{Western blot analysis}

Lysates from cells were obtained with lysis buffer. Proteins were loaded onto 10\% SDS-PAGE and transferred to PVDF membranes (Millipore, CA, USA). Blots were blocked with $5 \%$ fat-free dry milk for 2 $\mathrm{h}$ and incubated with a rabbit anti-ATG14, SM- $\alpha$ Actin, SM-MHC, MMP-2, TNF- $\alpha$ or $\beta$-actin antibody $(1: 500$, Santa Cruz Biotechnology, USA) overnight at $4^{\circ} \mathrm{C}$. Then, membranes were incubated with HRP-conjugated goat anti-rabbit IgG (1:6000, Cell Signaling Technology, USA) for $2 \mathrm{~h}$. The proteins were detected using ECL chemiluminescence.

\section{Statistical analysis}

Statistical analysis was performed using the SPSS 16.0 statistics software (SPSS, Chicago, IL). Data were expressed as the mean \pm SD. All data were statistically analysed using a one-way ANOVA followed by the Student-Newman-Keuls post hoc tests or Student's t-test. $P<0.05$ was considered statistically significant. 


\section{Results}

Expression of miR-29b and autophagy-related genes in the IA patients

The serum level of miR-29b was remarkably downregulated in the IA patients compared with that of the normal subjects. Moreover, the miR-29b level in the ruptured group was lower than that in the unruptured group (Fig. 1A). These results indicated that decreased miR-29b could be associated with IA formation and progression to rupture. In addition, we also found LC-3, Beclin-1, ATG5 and ATG14 were significantly increased and p62 was decreased in the aneurysm tissue of IA patients (Fig. 1B), suggesting that the autophagic process was activated in the occurrence and development of IA.

\section{Knockdown of miR-29b enhanced VSMCs proliferation and migration}

To explore the roles of miR-29b in VSMCs function, we infected VSMCs with LV-miR29b or LV-anti-miR-29b. The transfection efficiency of miR-29b was confirmed by real-time

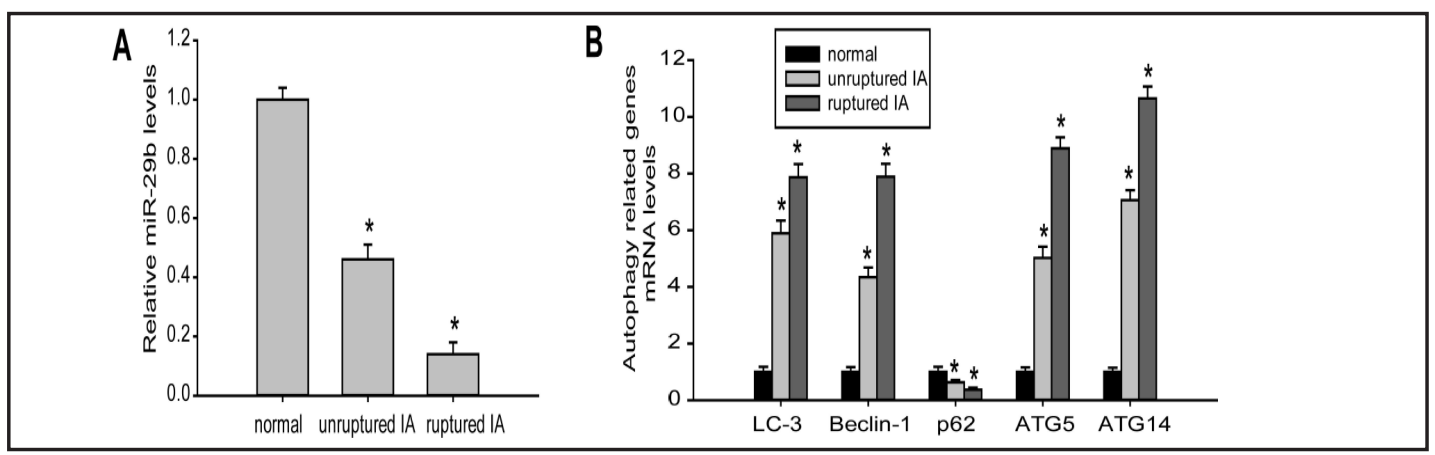

Fig. 1. The level of miR-29b and autophagy in IA patients. A: Serum level of miR-29b was significantly decreased in the unruptured and ruptured IA patients relative to that of normal subjects. B: Activated autophagy was confirmed in the aneurysm tissue of IA patients. The data are represented as the mean \pm SD. $* P<0.05$, compared to the normal group.

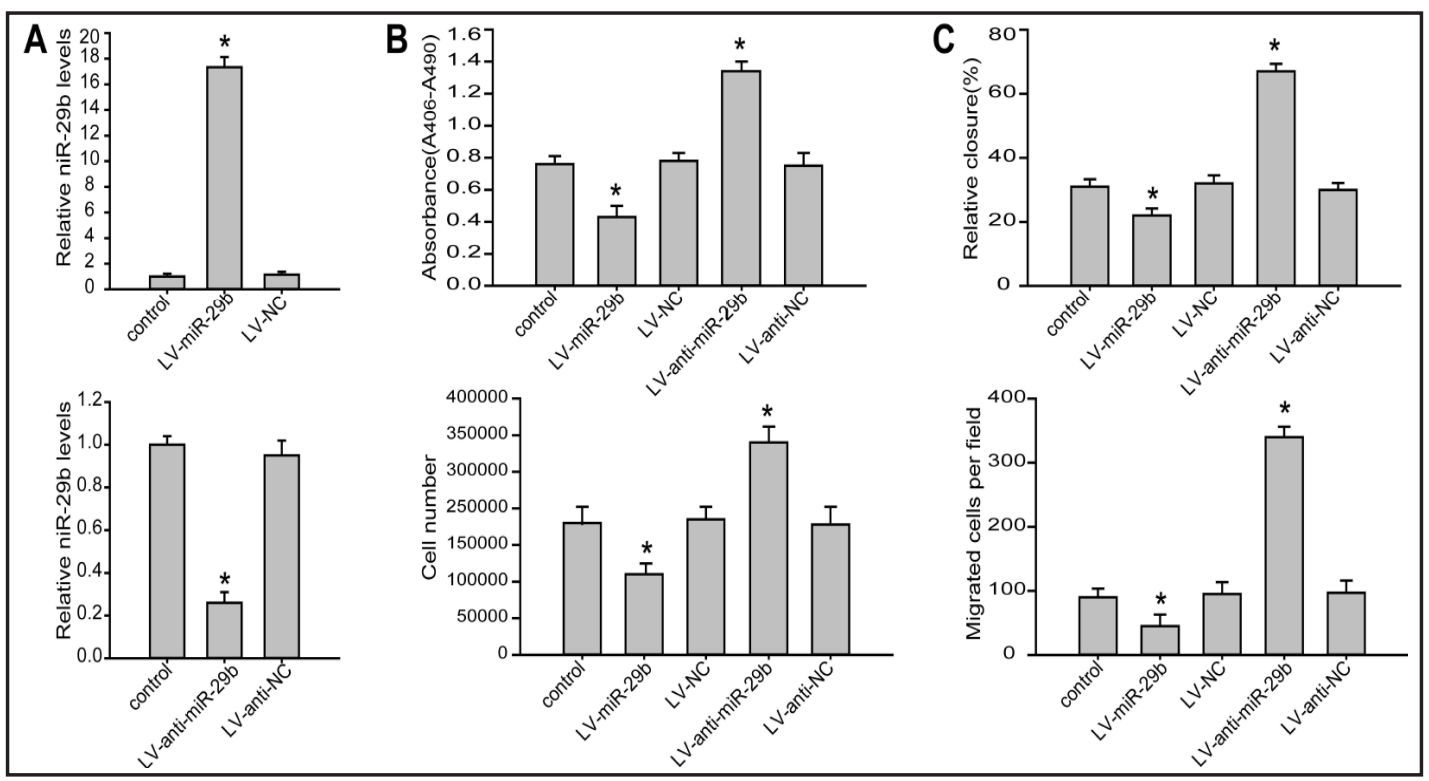

Fig. 2. miR-29b affected VSMCs proliferation and migration. A: The transfection efficiency of miR-27a in VSMCs was confirmed by real-time PCR. B: The effect of miR-29b on VSMCs proliferation was detected by BrdU assay and cell counting. C: The role miR-29b in VSMCs migration was evaluated by a wound-healing assay and transwell migration analysis. The data are represented as the mean \pm SD. $* P<0.05$, compared to the control group. 


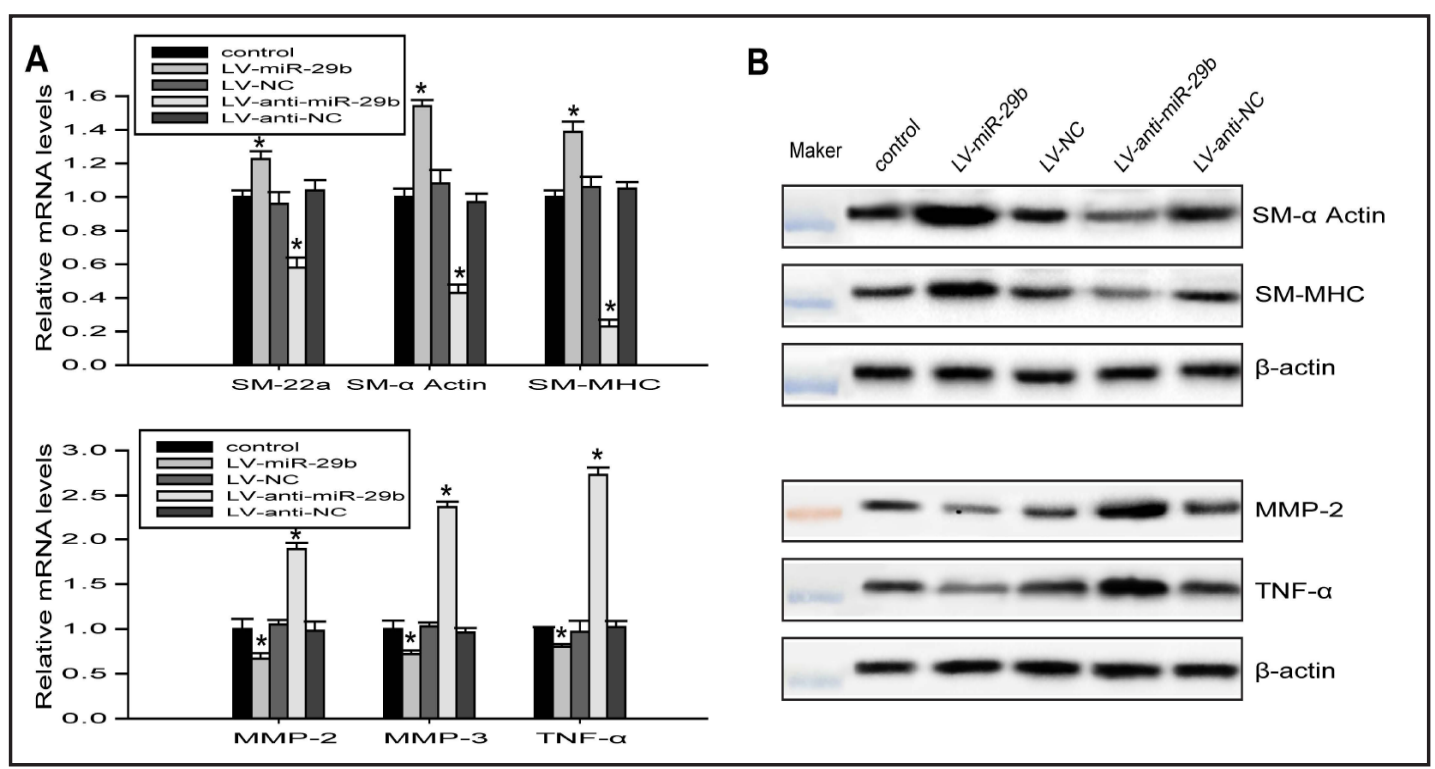

Fig. 3. The role of miR-29b in VSMCs phenotypic modulation. A: miR-29b was shown to affect the mRNA levels of contractile- and synthetic-specific indicators by real-time PCR. B: The protein expression of contractile- and synthetic-specific indicators was determined by Western blot. The data are represented as the mean \pm SD. ${ }^{*} P<0.05$, compared to the control group.

PCR. As shown in Figure 2A, the miR-29b level was significantly upregulated by LV-miR$29 \mathrm{~b}$ and downregulated by LV-anti-miR-29b. Knockdown of miR-29b significantly enhanced VSMCs proliferation as demonstrated by the BrdU incorporation assays and cell counting (Fig. 2B). Meanwhile, the migratory tendency of VSMCs transfected with LV-anti-miR-29b was significantly higher than that of their counterparts in the control group as demonstrated in the wound healing and transwell migration assays (Fig. 2C). In contrast, miR-29b overexpression significantly inhibited cell proliferation and migration.

\section{Knockdown of miR-29b induced VSMCs phenotypic modulation}

We examined the potential effect of miR-29b on phenotypic modulation in cultured VSMCs. We found that miR-29b knockdown significantly reduced expression of contractile proteins, such as smooth muscle myosin heavy chain (SM-MHC), SM-a-actin, and SM-22a, whereas it increased the expression of matrix metalloproteinase-2 (MMP-2), MMP-3 and tumour necrosis factor (TNF- $\alpha$ ). However, overexpression of miR-29b significantly prevented VSMCs phenotypic modulation (Fig. 3).

Knockdown of miR-29b promoted autophagy activation in VSMCs

Elevated LC3 expression was observed in the cytoplasm of cultured VSMCs transfected with LV-anti-miR-29b (Fig. 4A). Additionally, the mRNA levels of Beclin-1, ATG5 and ATG14 were significantly upregulated, and p62 was notably downregulated in the LV-anti-miR$29 \mathrm{~b}$ group. In contrast, the VSMCs exposed to LV-miR-29b showed reduced Beclin-1, ATG5 and ATG14 levels, as well as increased levels of p62 (Fig. 4B). Additionally, Western blot results showed that LC3II expression was increased and p62 level was decreased in VSMCs treated with LV-anti-miR-29b (Fig. 4C). These data indicated that miR-29b played a critical role in regulating the autophagic process.

\section{ATG14 was a potential target gene of miR-29b}

We sought to identify the potential target genes of miR-29b using TargetScan. Among them, ATG14, involved in the regulation of autophagy, was chosen as a candidate. The 3'UTR region of ATG14 mRNA has unique putative binding sites for miR-29b (Fig. 5A). 


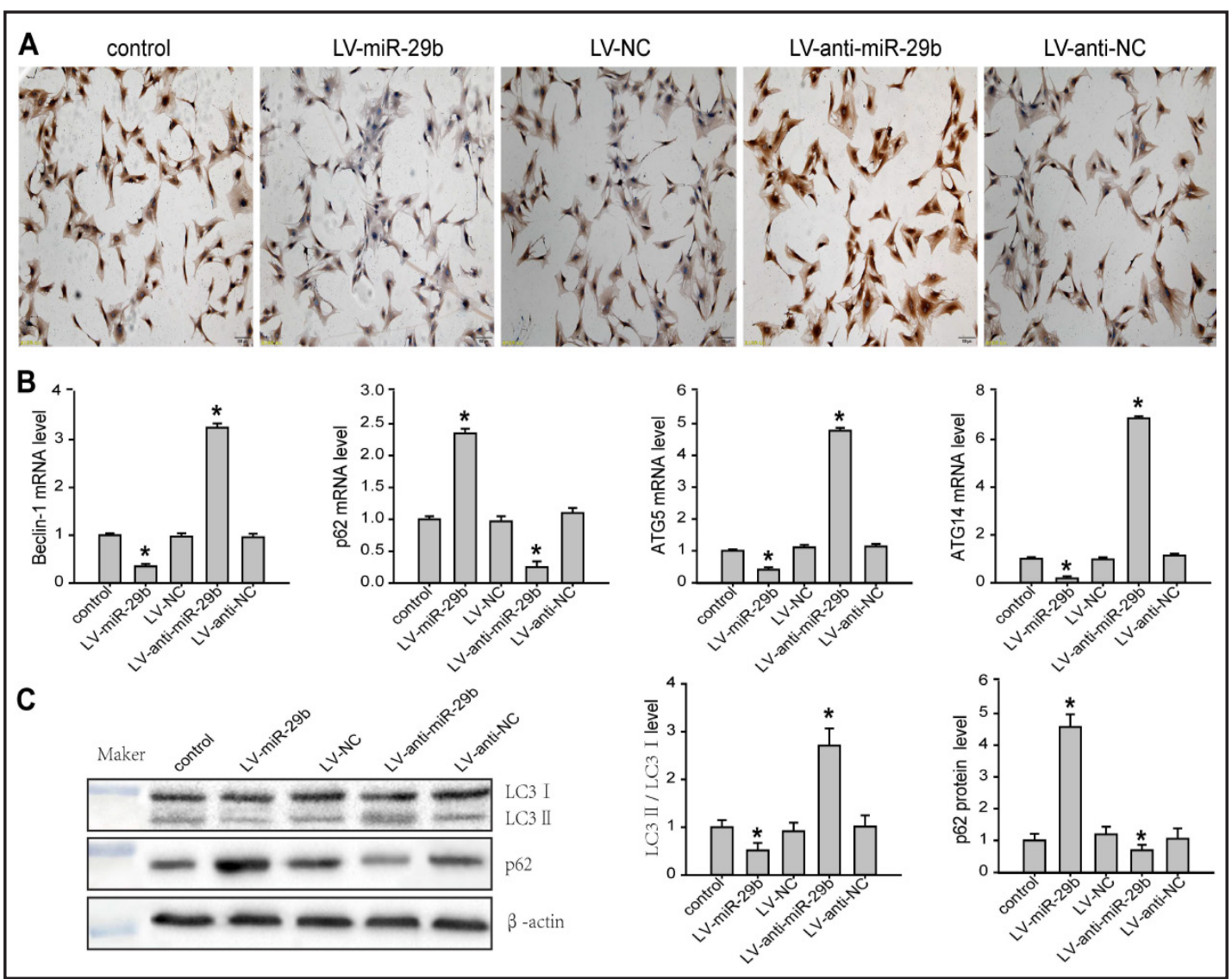

Fig. 4. The autophagic process was regulated by miR-29b in VSMCs. A: Representative immunocytochemical images of the expression of the LC3 protein in the VSMCs. B: The mRNA levels of Beclin-1, p62, ATG5 and ATG14 were analysed by real-time PCR. C: The protein expression of LC3 and p62 was evaluated by Western blot. The data are represented as the mean \pm SD. ${ }^{*} P<0.05$, compared to the control group.

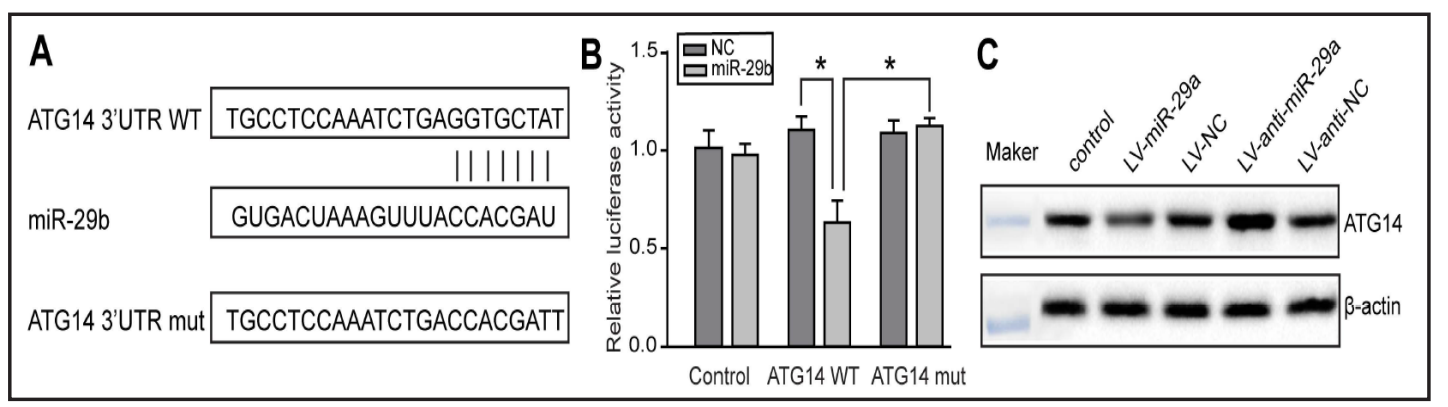

Fig. 5. ATG14 was a functional target gene of miR-29b. A: Sequence of the seed region from miR-29b and targeting sites from the 3' UTR of ATG14. B: The interaction between miR-29b and the 3'-UTR region of ATG14 mRNA was detected using a relative luciferase activity assay in the cultured VSMCs. C: Western blot analysis to quantify the protein level of ATG14 in VSMCs. The data are represented as the mean \pm SD. ${ }^{*} P<$ 0.05 , compared to the control group.

Furthermore, the luciferase reporter assay indicated that the relative luciferase activity was markedly decreased in the cells co-transfected with both ATG14-3'UTR WT and miR-29b, whereas there were no apparent changes in the cells co-transfected with ATG14-3'UTR mut and miR-29b (Fig. 5B). These results revealed that ATG14 was a direct target gene of miR$29 \mathrm{~b}$. Additionally, the results showed that miR-29b could negatively regulate the levels of ATG14 mRNA and protein in the cultured VSMCs (Fig. 5C and Fig. 4B).

\section{KARGER}




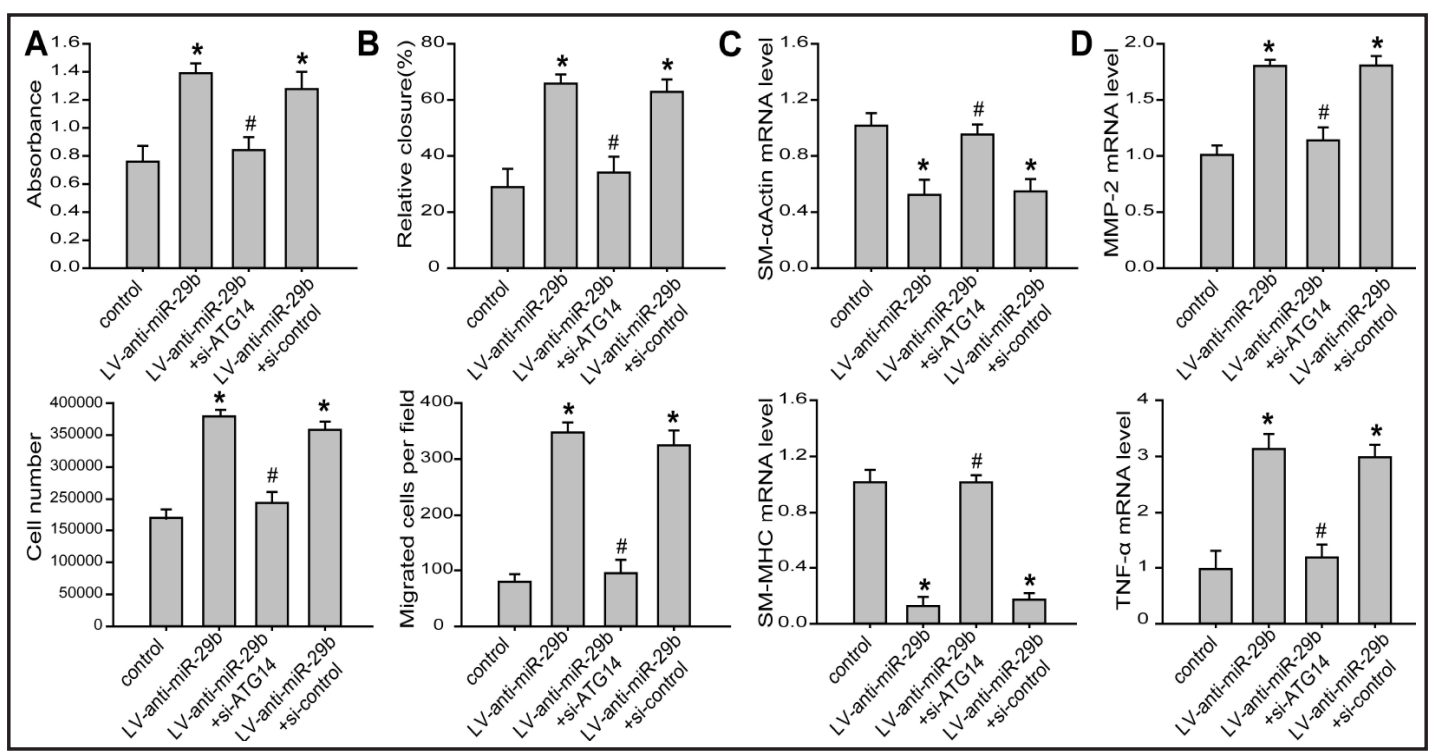

Fig. 6. Knockdown of ATG14 reversed the effects of miR-29b inhibition. A and B: Knockdown of ATG14 blocked the induction of proliferation and migration by miR-29b downregulation. C and D: Knockdown of ATG14 abrogated miR-29b downregulation-induced VSMCs phenotypic modulation. The data are represented as the mean $\pm \mathrm{SD}$. ${ }^{*} P<0.05$, compared to the control group, ${ }^{\#} P<0.05$, compared to the LV-anti-miR$29 b$ group.

Knockdown of ATG14 by siRNA reversed the effects of miR-29b inhibition

To further confirm whether the functional roles of miR-29b in VSMCs proliferation, migration and phenotypic modulation were dependent on ATG14, cultured VSMCs were co-transfected with si-ATG14 and LV-anti-miR-29b. These results suggested that the effects of miR-29b inhibition, including promoting proliferation, migration and phenotypic modulation, were partially reversed with co-transfection with si-ATG14 and LV-anti-miR-29b (Fig. 6). All data indicated that miR-29b knockdown induced VSMCs phenotypic modulation by enhancing ATG14-mediated autophagy.

\section{Discussion}

To date, except for surgical procedures, there is no available medical treatment to prevent the rupture of an IA. Given the severity and negative social impact of a resulting SAH after rupture, the mechanisms underlying IA formation and rupture should be investigated to develop a novel medical treatment for IA. In this study, we validated that the serum level of miR-29b was significantly decreased in the IA patients compared to that of normal subjects, indicating that miR-29b could be involved in the formation, progression, and rupture of IA. Importantly, miR-29b levels in the ruptured group were lower than that of the unruptured group, suggesting that miR-29b represented a warning molecule for IA rupture.

Next, we investigated the impact of miR-29b on VSMCs proliferation, migration and phenotypic modulation from a contractile to synthetic phenotype. VSMCs phenotypic modulation was known to be important in the pathogenesis of IA and other vascular diseases [11]. Our results showed that knockdown of miR-29b dramatically inhibited VSMCs proliferation and migration, while overexpression of miR-29b have the opposite effect. Furthermore, miR-29b knockout induced VSMCs phenotypic modulation, as evidenced by a decreased expression of contractile proteins, such as SM-22a, SM-aActin and SMMHC, and increased expression of matrix MMP-2, MMP-3 and TNF- $\alpha$, whereas miR-29b overexpression inhibited VSMCs phenotypic conversion. In most IA walls, VSMCs migrate to the intima, where they undergo phenotypic modulation from a contractile to synthetic 


\section{Cellular Physiology Cell Physiol Biochem 2017;41:510-518 \begin{tabular}{l|l|l} 
DOI: 10.1159/000456887 & and Biochemistry \\
Published onnine; January 30, 2017 & $\begin{array}{l}\text { O 2017 The Author(s). Published by S. Karger AG, Basel } \\
\text { www.karger.com/cpb }\end{array}$
\end{tabular} \\ Sun et al.: MiR-29b Downregulation Induces Phenotypic Modulation in VSMCs}

phenotype characterized by enhanced proliferation, migration, synthesis and secretion of ECM and pro-inflammatory cytokines [11], resulting in increased tensile strength of the continually degenerating IA wall. Therefore, modulation of VSMCs phenotypes is critical to the degeneration and ultimate rupture of IA walls. However, the molecular mechanism underlying the involvement of miR-29b in VSMCs phenotypic modulation is unclear.

Autophagy, a self-degradative lysosomal-mediated process, plays an important role in maintaining cellular homeostasis [12]. Autophagy is involved in numerous vascular disease states, including atherosclerosis, hypertension and restenosis [13]. Interestingly, an enhanced level of autophagy was observed in the aneurysm tissue of IA patients, especially in patients with ruptured aneurysms. An increasing number of studies have suggested that autophagy plays a critical role in the modulation of VSMCs phenotype and viability [14]. Autophagy initiated by PDGF has been shown to remove contractile proteins by lipid electrophiles [15]; thus, this form of autophagy could promote the transition to synthetic VSMCs and increase cell survival. Therefore, inhibition of autophagy could prevent phenotypic changes [16].

Additionally, the oscillation of VSMCs between a contractile and synthetic phenotype was dependent on functional miRNAs. miR-1 was reported to upregulate the expression of VSMC-specific contractile proteins by directly targeting KLF4 [17]. Notably, the transfer of miR-143 and miR-145 from endothelial cells to VSMCs via microvesicles promoted a contractile VSMCs phenotype [18]. Our results demonstrated that inhibition of miR-29b not only induced VSMCs phenotypic modulation but also enhanced autophagy activation. Furthermore, we tested the hypothesis that the effect of miR-29b on VSMCs phenotypic modulation might be associated with the autophagy process. In this study, bioinformatics analysis suggested that ATG14 was a potential target gene of miR-29b. ATG14, required for autophagosome biogenesis, is a critical switch for the temporal and spatial control of autophagic membrane fusion [19]. We have found that the expression levels of miR-29b and ATG14 display a notable negative correlation in the IA patients. We also identified that miR$29 \mathrm{~b}$ could directly bind to the 3' UTR region of ATG14 mRNA using a luciferase reporter system. Moreover, miR-27a negatively regulated the mRNA and protein levels of ATG14 in VSMCs. Notably, data from co-transfection experiments revealed that silencing of ATG14 by siRNA apparently abrogated the miR-29b knockdown-mediated phenotypic modulation.

In summary, we have demonstrated that miR-29b was decreased in both the unruptured and ruptured IA patients. miR-29b knockdown stimulated VSMCs and resulted in VSMCs switching from the "contractile" phenotype to the "synthetic" phenotype associated with the induction of proliferation and migration in vitro. More importantly, miR-29b knockdown induced VSMCs phenotypic modulation through the modulation of ATG14-mediated autophagy. This study will provide the basis for the development of therapeutic targets in the prevention of IA progression to rupture.

\section{Acknowledgements}

This work was supported by a grant from the China Postdoctoral Science Foundation (2015M580117), and the Project supported by the Beijing Postdoctoral Research Foundation (2015ZZ-49 and 2016ZZ-38).

\section{Disclosure Statement}

The authors have no conflicts to disclose.

\section{References}

1 Rinkel GJE: Natural history, epidemiology and screening of unruptured intracranial aneurysms. J Neuroradiol 2008;35:99-103. 


\section{Cellular Physiology Cell Physiol Biochem 2017;41:510-518

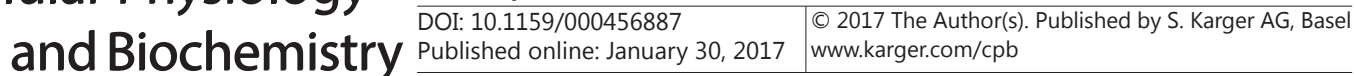

- Kataoka H: Molecular mechanisms of the formation and progression of intracranial aneurysms. Neurol Med Chir (Tokyo) 2015;55:214-229.

3 Frösen J: Smooth muscle cells and the formation, degeneration, and rupture of saccular intracranial aneurysm wall--a review of current pathophysiological knowledge. Transl Stroke Res 2014;5:347-356.

4 Starke RM, Chalouhi N, Ding D, Raper DM, Mckisic MS, Owens GK, Hasan DM, Medel R, Dumont AS: Vascular smooth muscle cells in cerebral aneurysm pathogenesis. Translational Stroke Research 2014;5:338-346.

5 Chen X, Zhao Y, Wang F, Bei Y, Xiao J, Yang C: MicroRNAs in Liver Regeneration. Cell Physiol Biochem 2015;37:615-628.

-6 Jin H, Li C, Ge H, Jiang Y, Li Y: Circulating microRNA: a novel potential biomarker for early diagnosis of Intracranial Aneurysm Rupture a case control study. J Transl Med 2013;11:1-9.

7 Albinsson S, Sessa WC: Can microRNAs control vascular smooth muscle phenotypic modulation and the response to injury? Physiol Genomics 2011;43:529-533.

-8 Cheng Y, Liu X, Jian Y, Ying L, Xu DZ, Qi L, Deitch EA, Huo Y, Delphin ES, Zhang C: MicroRNA-145, a novel smooth muscle cell phenotypic marker and modulator, controls vascular neointimal lesion formation. Circ Res 2009;105:158-166.

-9 Chistiakov DA, Sobenin IA, Orekhov AN, Bobryshev YV: Human miR-221/222 in Physiological and Atherosclerotic Vascular Remodeling. Biomed Res Int 2015;2015:354517.

10 Xiao Q Chen Q, Feng Y: miRNA-34a reduces neointima formation through inhibiting smooth muscle cell proliferation and migration. J Mol Cell Cardiol 2015;101:A116-A116.

-11 Ali MS, Starke RM, Jabbour PM, Tjoumakaris SI, Gonzalez LF, Rosenwasser RH, Owens GK, Koch WJ, Greig NH, Dumont AS: TNF- $\alpha$ induces phenotypic modulation in cerebral vascular smooth muscle cells: implications for cerebral aneurysm pathology. J Cereb Blood Flow Metab 2013;33:1564-1573.

12 Cheng Y, Wang B, Zhou H, Dang S, Jin M, Shi Y, Hao L, Yang Z, Zhang Y: Autophagy is Required for the Maintenance of Liver Progenitor Cell Functionality. Cell Physiol Biochem 2015;36:1163-1174.

13 De Meyer GR, Grootaert MO, Michiels CF, Kurdi A, Schrijvers DM, Martinet W: Autophagy in Vascular Disease. Circ Res 2015;116:468-479.

14 Salabei JK, Hill BG: Autophagic regulation of smooth muscle cell biology. Redox Biol 2014;4:97-103.

15 Salabei JK, Cummins TD, Singh M, Jones SP, Bhatnagar A, Hill BG: PDGF-mediated autophagy regulates vascular smooth muscle cell phenotype and resistance to oxidative stress. Biochem J 2013;451:375-388.

16 Li BH, Liao SQ, Yin YW, Long CY, Guo L, Cao XJ, Liu Y, Zhou Y, Gao CY, Zhang LL: Telmisartan-induced PPAR $\gamma$ activity attenuates lipid accumulation in VSMCs via induction of autophagy. Mol Biol Rep 2015;42:179186.

17 Xie C, Huang H, Sun X, Guo Y, Hamblin M, Ritchie RP, Garciabarrio MT, Zhang J, Chen YE: MicroRNA-1 regulates smooth muscle cell differentiation by repressing Kruppel-like factor 4. Stem Cells Dev 2011;20:205-210.

18 Hergenreider E, Boon RA, Heydt S, Tréguer K, Böttger T, Horrevoets AJ, Zeiher AM, Braun T, Urbich C, Dimmeler S: Abstract 11676: Atheroprotective Communication Between Endothelial Cells and Smooth Muscle Cells via KLF2-Dependent Enrichment of miRNAs in Microvesicles. Circulation 2011;124:A11676.

19 Diao J, Liu R, Rong Y, Zhao M, Zhang J, Lai Y, Zhou Q, Wilz LM, Li J, Vivona S: ATG14 promotes membrane tethering and fusion of autophagosomes to endolysosomes. Nature 2015;520:563-566. 